\title{
Ladybird beetles fauna (Coleoptera: Coccinellidae) of the Republic of Mordovia, Russia
}

\author{
ALEXANDER B. RUCHIN ${ }^{1, \vartheta}$, LEONID V. EGOROV ${ }^{1,2, v \bullet}$, GENNADY B. SEMISHIN $^{1}$ \\ ${ }^{1}$ Joint Directorate of the Mordovia State Nature Reserve and National Park "Smolny". Saransk, Dachnyi Lane, 4, 430011, Russia. \\ "email: sasha_ruchin@rambler.ru \\ ${ }^{2}$ State Nature Reserve «Prisursky», Lesnoi, 9, Cheboksary, 428034, Russia. ^"email: platyscelis@ mail.ru
}

Manuscript received: 25 December 2018. Revision accepted: 8 January 2019.

\begin{abstract}
Ruchin AB, Egorov LV, Semishin GB. 2019. Ladybird beetles fauna (Coleoptera: Coccinellidae) of the Republic of Mordovia, Russia. Biodiversitas 20: 316-327. The paper presents the first overview of Coccinellidae fauna of the Republic of Mordovia, Russia. Nowadays 46 species are reliably known in Mordovia. Of these, three species have firstly been registered in the Republic of Mordovia in recent years, namely Nephus bipunctatus, Hyperaspis erythrocephala, and Oenopia conglobata. In addition, three species were recommended to be excluded from the list of regional fauna.
\end{abstract}

Keywords: Coccinellidae, Coleoptera, distribution, biology, population, ladybird beetles, Russia, Republic of Mordovia

\section{INTRODUCTION}

Biodiversity is a data-intense science, drawing as it does on data from a large number of disciplines in order to build up a coherent picture of the extent and trajectory of life on earth (Grebennikov 2016). The Republic of Mordovia is located in the center of the East European Plain in the south-western periphery of the Volga basin in the interfluve of Moksha river and Sura river (Ruchin et al. 2018). Therefore, it is expected that region's biodiversity should be high enough. In the last decade as a result of invertebrate fauna inventory, 4000 species were found in the Republic of Mordovia for the first time (Ruchin and Kurmaeva 2010; Chikhlyaev and Ruchin 2014; Chikhlyaev et al. 2016; Ruchin and Artaev 2016; Egorov and Shapovalov 2017; Ruchin and Makarkin 2017; Chursina and Ruchin 2018a, 2018b; Ruchin 2018; Ruchin and Egorov 2017, 2018a, 2018b, 2018c; Ruchin and Grishutkin 2018; Ruchin and Mikhailenko 2018; Ruchin et al. 2018, 2019; Tomaszewska et al. 2018). In doing so, some of these findings are rare species. These findings are of great interest for the researchers.

Coccinellidae (Coleoptera) is a relationally nonnumerous family. It consists of about 6000 species in the world fauna (Bouchard et al. 2017); while in Russia more than 160 species are known (Iablokoff-Khnzorian 1983; Korotyaev and Lobanov 2019). The Coccinellidae is one of the most useful in practice among Coleoptera families. Some species of ladybird beetles can regulate the abundance of populations of Cimicidae, Aphidoidea, Coccoidea (Delucchi 1954; Booth 1991; Cheah and McClure 2000; Iftikhar et al. 2018), as well as members of Aleyrodidae, Thripidae (Satti and Mahgoub 2018) and many other soft-bodied insect pests. Therefore, it is widely used for plant protection as a part of biological methods (Evans 1991; Félix and Soares 2004; Soares and Serpa
2007; Khan and Yoldas 2018). On the other hand, few Coccinellidae species are considered as invasive beetles causing visible damage to agriculture (Kajita et al. 2000; Mizell 2007; Soares et al. 2008; Orlova-Bienkowskaja and Bieńkowski 2017).

To date, Coccinellidae fauna of the Middle Volga Region consists of about 90 species. The Mordovia State Nature Reserve staff has started the research of Coccinellidae fauna in the Republic of Mordovia. So, Plavilshchikov (1964) has registered 15 species of Coccinellidae species in the Mordovia State Nature Reserve. Later Timraleev (1989) has noted 19 species from 16 genera in the Republic of Mordovia. But the author didn't take into account the article of Plavilshchikov (1964). The relations between Coccinellidae and their potential prey have been studied in agrocoenoses (Antsiferova et al. 1966; Antsiferova and Dobrosmyslov 1966; Antsiferova 1979). Ten Coccinellidae species have been registered on cereal crops (Timraleev 1992). According to the latest data of Timraleev et al. (2007), 20 Coccinellidae species have been found in Mordovia. The main part of these results has already been published (Ruchin 2015; Egorov and Ruchin 2009a). This study was aimed to generalize data on Coccinellidae fauna in the Republic of Mordovia.

\section{MATERIALS AND METHODS}

The study has been carried out during 2003-2018 (most intensively in 2008-2018). We used the generally accepted entomological field methods (Fasulati 1971). Finally, more than 4300 specimens were examined.

The description of each species in the annotated list below includes following data: (i) literature devoted to a species within the study area (if it contains a reliable 
location of species' record); (ii) newly found locations which have not been published before; (iii) date of collecting; (iv) number of collected specimens; (v) full name of a collector; (vi) notes (if any). If species' location has been found by authors, full name of a collector is not mentioned. The following information is written separately: biology of a species in the Republic of Mordovia, characteristics of habitats preferred by a Coccinellidae species; selected observations of the species' phenology. Species newly registered in Mordovia are marked by asterisk (*). Dash «-» is used to mark the species excluded from the list of regional fauna for different reasons (mentioned to Remarks). The question mark «?» is used for species whose presence in Mordovia needs to be proved by modern data.

Nomenclature of Coccinellidae species is used according to the last edition of Catalogue of Palaearctic Coleoptera (Kováŕ 2007). Specimen collections are stored in Zoological Museum of Moscow University (Moscow), Zoological Institute of the Russian Academy of Sciences (ZIN) (Saint Petersburg), Museum of the Mordovia State Nature Reserve (Pushta), Biological Museum of the Mordovia State University (Saransk) and private collections. There are abbreviations used in the article: MSNR - Mordovia State Nature Reserve, NPS - National Park «Smolny», exemplar - ex.

\section{Coccinellidae Latreille, 1807}

\section{Epilachninae Mulsant, 1846}

Subcoccinella vigintiquatuorpunctata (Linnaeus, 1758)

Literature. Temnikov Distr. (Ruchin et al. 2009a; Feoktistov 2011; Egorov and Ruchin 2013, 2014; Egorov et al. 2015, 2017). Ichalki Distr. (Ruchin et al. 2007b; Ruchin 2008b).

Materials. Tengushevo Distr., Khlebino, 18.VII.2015, 1 ex. Temnikov Distr., MSNR, quarter 442, 3.VI.2017, 1 ex.; MSNR, quarter 368, 27.V.2018, 1 ex.; MSNR, quarter 19, 8.VII.2018, 1 ex. Krasnoslobodsk Distr., Penkovo, 31.VIII.2008, 1 ex. Ruzaevka Distr., Levzhenskiy, 22.VII.2008, 1 ex. Atyashevo Distr., Selishchi, 24.VI.2016, 1 ex. Dubenki Distr., 8 km SW Yengalychevo, 1.VII.2009, 1 ex. Kochkurovo Distr., Mordovskoe Davydovo, 23.VII.2008, 1 ex., Kurmaeva D.K. Saransk, 25.VII.2006, 1 ex.

Habitat. It is known on steppe slopes, in mixed and deciduous forests.

\section{Coccinellinae Latreille, 1807}

\section{Anisosticta novemdecimpunctata (Linnaeus, 1758)}

Literature. Temnikov Distr. (Plavilshchikov 1964; Ruchin et al. 2009a; Egorov and Ruchin 2012, 2013, 2014; Egorov et al. 2015, 2016, 2017).

Materials. Temnikov Distr., MSNR, cordon Dolgiy Most, quarter 408, 13.V.2017, 1 ex., Egorov L.V.; MSNR, quarter 37, 16.V.2018, Egorov L.V.; MSNR, quarter 368, 27.V.2018, 3 ex.; quarter 368, 18.VI.2018, 2 ex., Semishin
G.B. Bolshie Berezniki Distr., 9 km S Simkino, 1.VI.2004, 1 ex.

Habitat. It is known in wet biotopes: on river banks, lake shores, in mature pine sphagnum forests with participation of Picea and Betula, near forest puddles.

Coccinula quatuordecimpustulata (Linnaeus, 1758) (Figure 1)

Literature. Temnikov Distr. (Plavilshchikov 1964; Ruchin et al. 2009a; Egorov and Ruchin 2012, 2013, 2014; Egorov et al. 2015, 2016, 2017). Ichalki Distr. (Ruchin et al. 2007b; Ruchin 2008b). Romodanovo Distr., Bolshie Berezniki Distr. (Antsiferova 1979).

Materials. Tengushevo Distr., $6 \mathrm{~km}$ west of Barashevo, 18.VII.2015, 1 ex.; Barashevo, 3.V.2008, 1 ex. Temnikov Distr., Temnikov, 1.VI.2008, 1 ex.; Staryi Gorod, 17.VIII.2008, 1 ex., Kurmaeva D.K.; Alkaevo, 15.VIII.2015, 1 ex.; Polyanki, 24.VII.2016, 1 ex.; MSNR, quarter 424, 13.V.2017, 1 ex.; MSNR, cordon Inorskiy, 13.V.2017, 1 ex., Egorov L.V., 23-24.VIII.2017, 2 ex., Semishin G.B.; MSNR, quarter 436, 1-15.VI.2017, 1 ex., Egorov L.V., Semishin G.B.; MSNR, quarter 442, 3.VI.2017, 1 ex., MSNR, cordon Drozhdenovkiy, 4.VI.2017, 2 ex.; MSNR, quarter 324, 13.VII.2017, 1 ex.; MSNR, cordon Podrubnyi, 16.VII.2017, 1 ex.; MSNR, quarter 86, 20.IV.2018, 1 ex., Semishin G.B.; MSNR, Pushta, 1.V.2018, 1 ex.; MSNR, quarter 368, 18.V.2018, 27.V.2018, 2 ex., Egorov L.V.; MSNR, quarter 330, 26.V.2018, 1 ex.; MSNR, quarter 332, 23.VI.2018, 1 ex.; quarter 19, 8.VII.2018, 1 ex. Zubova Polyana Distr., Udevo, 8.VI.2008, 1 ex.; 8 km SW Vysha, 31.VII.2009, 1 ex.; Tenishevo, 2.VIII.2015, 1 ex.; Lesnoy, 2.VIII.2015, 1 ex. Aturyevo Distr., Strelnikovo, 2.VII.2016, 2 ex.; Pichepolonga, 2.VII.2016, 1 ex. Torbeevo Distr., Drakino, 26.VII.2016, 2 ex. Elniki Distr., Malye Mordovskie Poshaty, 19.VII.2015, 23.VII.2016, 4 ex.; Novye Shaly, 19.VII.2015, 22.VII.2017, 3 ex.; Svobodnyi, 31.VII.2008, 6 ex. Krasnoslobodsk Distr., Staroe Sindrovo, 1.VIII.2008, 2 ex.; Penkovo, 31.VIII.2008, 3 ex.; Krasnoslobodsk, 8.V.2009, 1 ex.; 5 km NE of Staraya Avgura, 8.VII.2017, 1 ex. Kovylkino Distr., Chepurnovka, 29.VI.2017, 2 ex. Staroe Shaygovo Distr., Staroe Akshino, 11.V.2008, 16.VIII.2008, 30.IV.2017, 4 ex.; Lesnichestvo, 17.V.2008, 1 ex.; Ingener-Pyatina, 10.VII.2016, 1 ex. Ichalki Distr., NPS, Barakhmanovskoe forestry, quarter 108, 20.VI.4.VII.2017, 1 ex., Semishin G.B., quarter 74, 6.VI.2017, 12.IX.2017, 13.IX.2017, 5 ex., Semishin G.B.; NPS, Kemlyanskoe forestry, quarter 22, 11.VIII.2018, 1 ex., Ручин А.Б.; quarter 105, 12.VII.2018, 2 ex. Ichalki Distr., Sosnovka, 6.VIII.2008, 2 ex.; Khanineevka, 23.VII.2008, 2 ex.; NPS, Lvovskoe forestry, Obrezki, 12.VII.2006, 27.VI.2008, IV-V.2008, IV-V.2008, 21.VI.2008, 6 ex. Romodanovo Distr., Pushkino, 17.VIII.2017, 1 ex.; Zarechnyi, 3.VIII.2018, 3 ex. Lyambir Distr., Atemar, 24.VI.2017, 7.V.2016, 2 ex.; Novaya Uda, 29.VII.2016, 2 ex. Insar Distr., Verkhnyaya Lukhma, 19.VIII.2017, 2 ex. Ardatov Distr., Sosnovoe, 18.VIII.2008, 1 ex. Dubenki Distr., Yavleyka, IV.2008, 10.VIII.2017, 4 ex.; Krasnye Luga, 11.VI.2017, 1 ex.; Olevka, 26.V.2017, 2 ex.; Oktyabrskiy, 26.V.2017, 1 ex.; Bolshoe Kuzmino, 
26.V.2017, 1 ex. Chamzinka Distr., Komsomolskiy, VIII.2009, 1 ex., Ryzhov M.K.; Gorbunovka, 24.VI.2016, 1 ex.; Mokshalei, 28.VII.2018, 1 ex. Atyashevo Distr., Atyashevo, 24.VI.2016, 1 ex.; Selishchi, 10.VIII.2017, 2 ex. Ruzaevka Distr., $3 \mathrm{~km} \mathrm{~N}$ of Streletskaya Sloboda, 19.V.2009, 1 ex.; Levzhenskiy, 15.V.2007, 13.VI.2015, 28.VII.2016, 4 ex., 22.VII.2008, 1 ex. Bolshie Berezniki Distr., 9 km S Simkino, 6.VI.2007, 1 ex.; Gart, 19.VII.2008, 2 ex.; Simkino, 12.VIII.2017, 2 ex.; Simkinskoe lesnichestvo, 12.VIII.2017, 1 ex. Kochkurovo Distr., Starye Turdaki, 12.VI.2008, 2 ex.; Sabaevo, 3.IX.2009, 14.VII.2018, 3 ex.; Kachelay, 3.IX.2009, 3 ex.; Podlesnaya Tavla, 8.V.2016, 1 ex.; Kochkurovo, 8.V.2016, 2 ex.; Novaya Pyrma, 11.VIII.2017, 1 ex.; Krasnaya Zorka, 29.VII.2017, 1 ex. Saransk, 28.VI.2006, 22.IV.2008, 12.V.2009, 27.VIII.2009, 5 ex.

Habitat. It is known in different open habitats, predominantly in steppe slopes and dry grasslands.
- Coccinula sinuatomarginata (Faldermann, 1837)

Remarks. It is noted by Timraleev (1998) and Timraleev et al. (2007) without indication of localities. It inhabits dry steppes, predominantly cretaceous ones (Isaev and Egorov 2006). This species is not presented in the materials. Its presence in Mordovia is highly unreliable. Hence we recommended to exclude this species from the list of regional fauna.

\section{Tytthaspis gebleri (Mulsant 1850)}

Literature. Temnikov Distr. (Egorov and Ruchin 2013; Egorov et al. 2016).

Materials. Temnikov Distr., MSNR, cordon Dolgiy Most, 13.V.2017, 1 ex., Egorov L.V. Zubova Polyana Distr., 8 km SW Vysha, 31.VII.2009, 1 ex.; Tenishevo, 2.VIII.2015, 1 ex. Elniki Distr., Malye Mordovskie Poshaty, 19.VII.2015, 1 ex. Atyashevo Distr., Kamenka, 24.VI.2016, 1 ex.

Habitat. It is known in pine forest edges, dry grasslands, steppe slopes.

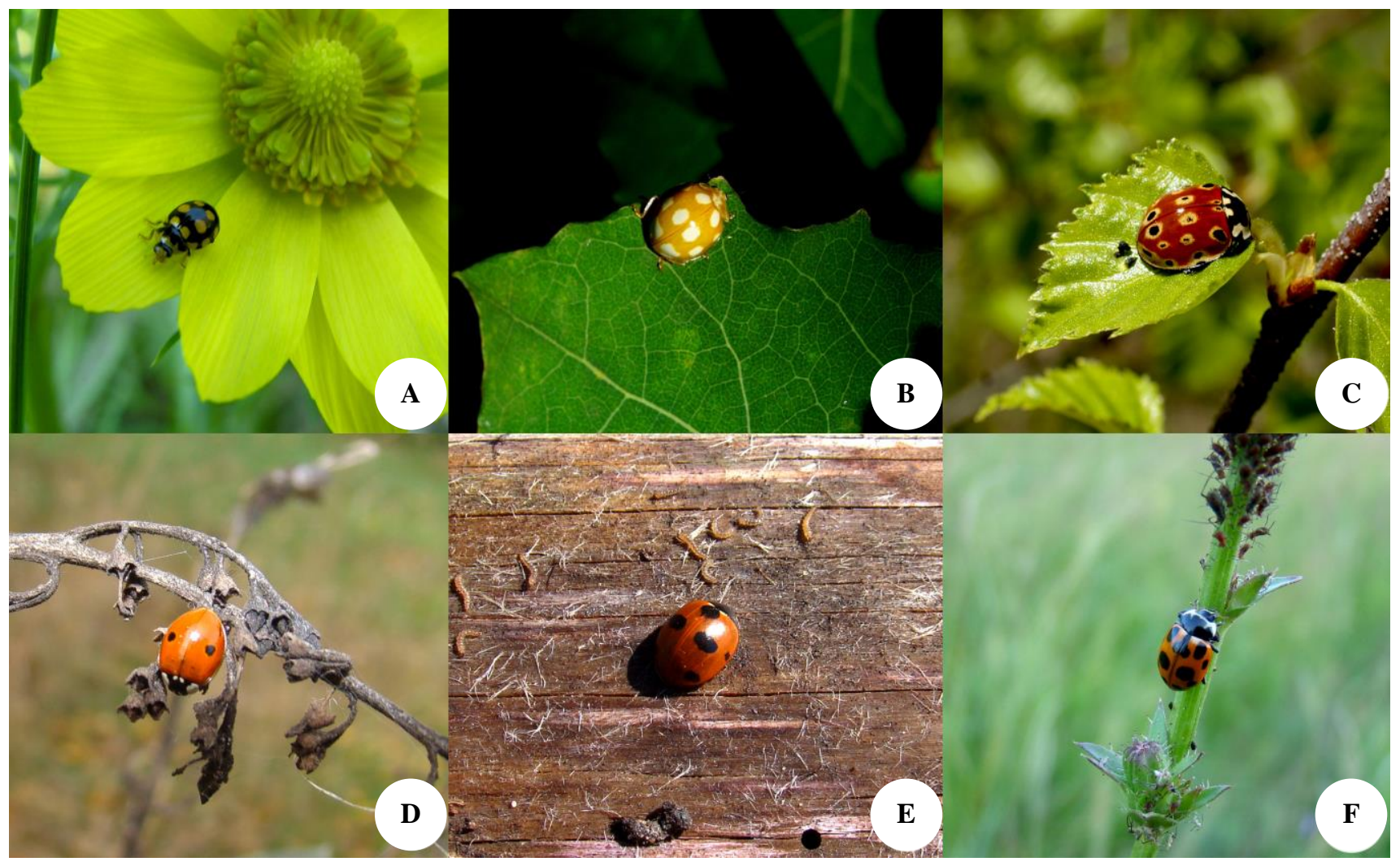

Figure 2. Photo of ladybirds beetles. A. Coccinula quatuordecimpustulata (Ruzaevka Distr., Levzhenskiy, photograph by A.B. Ruchin); B. Calvia decemguttata (Temnikov Dist., MSNR, cordon Inorskiy, photograph by G.B. Semishin); C. Anatis ocellata (Temnikov Dist., MSNR, cordon Pavlovskiy, photograph by G.B. Semishin); D. Adalia bipunctata (Ichalki Distr., NPS, Kemlyanskoe forestry, photograph by A.B. Ruchin); E. Coccinella magnifica (Temnikov Dist., MSNR, Pushta, photograph by A.B. Ruchin); F. Ceratomegilla notata (Krasnoslobodsk Distr., Staroe Sindrovo, photograph by A.B. Ruchin) 
Tytthaspis sedecimpunctata (Linnaeus, 1760)

Literature. Temnikov Distr. (Egorov and Ruchin 2013; Egorov et al. 2015, 2016, 2017). Ichalki Distr. (Ruchin 2008b).

Materials. Tengushevo Distr., $6 \mathrm{~km} \mathrm{~W}$ Barashevo, 18.VII.2015, 1 ex. Temnikov Distr., Tarkhany, 10.V.2009, 1 ex. Zubova Polyana Distr., 8 km SW Vysha, 31.VII.2009, 1 ex. Elniki Distr., Malye Mordovskie Poshaty, 19.VII.2015, 1 ex. Bolshoe Ignatovo Distr., NPS, Aleksandrovskoe forestry, Lesnoy, V.2007, 1 ex. Ardatov Distr., Olevka, 26.V.2017, 1 ex. Insar Distr., Verkhnyaya Lukhma, 19.VIII.2017, 1 ex. Lyambir Distr., Ekaterinovka, V.2007, 1 ex. Ruzaevka Distr., 3 km N Streletskaya Sloboda, V-VI.2009, 1 ex. Bolshie Berezniki Distr., Simkino, 9.VII.2009, 1 ex. Kochkurovo Distr., Sabaevo, 3.IX.2009, 1 ex.; Starye Turdaki, 8.IX.2009, 1 ex.

Habitat. It is known in floodplain meadows and steppe slopes, oak (Quercus) forest edges, mixed forest glades and spruce forests with participation of alder (Alnus) and birch (Betula), along the streams.

\section{Calvia decemguttata (Linnaeus, 1767) (Figure 1)}

Literature. Temnikov Distr. (Plavilshchikov 1964; Egorov and Ruchin 2012, 2014; Egorov et al. 2015, 2016, 2017). Ichalki Distr. (Egorov and Ruchin 2009).

Materials. Temnikov Distr., MSNR, quarter 442, 3.VI.2017, 1 ex.; MSNR, quarter 19, 15-16.V.2018, 18 ex., Egorov L.V.; MSNR, quarter 35, 16.V.2018, 1 ex., Egorov L.V.; MSNR, quarter 434, 18.VI.2018, 1 ex., Semishin G.B. Ichalki Distr., NPS, Barakhmanovskoe forestry, quarter 93, 1.VIII.2017, 1 ex., Semishin G.B. Ardatov Distr., Kozlovka, 2007, in spider-web on the electrical station, 2 ex. Lyambir Distr., Ekaterinovka, 29.V.2007, 1 ex. Saransk, 21.VIII.2008, 12.V.2009, 2 ex.

Habitat. It is known in forests of different types: mixed, deciduous, pine forests, forest plantations. The species individuals fly to the light.

\section{Calvia quatuordecimguttata (Linnaeus, 1758)}

Literature. Temnikov Distr. (Ruchin et al. 2009a; Feoktistov 2011; Egorov and Ruchin 2012, 2013, 2014; Egorov et al. 2015, 2016, 2017).

Materials. Temnikov Distr., Tatarskoe Karaevo, 20.IX.2008， 16.IV.2017, 2 ex.; MSNR, quarter 436, 2.VI.2017, 1 ex., Semishin G.B.; cordon Podrubnyi, 16.VII.2017, 1 ex.; cordon Inorskiy, 26.VII.2017, 1 ex., Semishin G.B.; MSNR, Pushta, 1.V.2018, 1 ex., Ручин A.Б.; MSNR, quarter 19, 15.V.2018, 27.VI.-12.VII.2018, 2 ex., Semishin G.B., Egorov L.V.; MSNR, quarter 86, 17.V.2018, 3 ex., Egorov L.V.; MSNR, quarter 349, 23.VI.2018, 1 ex.; MSNR, quarter 37, 8.VII.2018, 1 ex. Zubova Polyana Distr., Tenishevo, 2.V.2008, 1 ex. Krasnoslobodsk Distr., Penkovo, 31.VIII.2008, 1 ex. Ichalki Distr., NPS, Barakhmanovskoe forestry, quarter 112, 28.VIII.-19.IX.2017, 1 ex., Semishin G.B. Bolshie Berezniki Distr., $5 \mathrm{~km}$ SE Permisi, 12.VI.2015, 2 ex. Saransk, 6.VII.2007, 1 ex.

Habitat. As a rule, it inhabits forest associations: mature pine forests with participation of spruce, birch; deciduous forests; floodplain oak forests. In addition, it is observed along shores of water bodies, in floodplain meadows. It has been caught on inflorescences of Aegopodium podagraria L., on Padus avium Mill., leaves of Alnus glutinosa, Salix sp., Urtica sp.

\section{Calvia quindecimguttata (Fabricius, 1777)}

Literature. Temnikov Distr. (Egorov et al. 2015, 2016).

Habitat. Two specimens were found along the lakeshore in the Mordovia State Nature Reserve.

\section{Propylea quatuordecimpunctata (Linnaeus, 1758)}

Literature. Temnikov Distr. (Plavilshchikov 1964; Ruchin et al. 2009a; Egorov and Ruchin 2012, 2013, 2014; Egorov et al. 2015, 2016, 2017). Romodanovo Distr., Ruzaevka Distr., Bolshie Berezniki Distr. (Antsiferova et al. 1966; Antsiferova 1979). Ichalki Distr. (Ruchin et al. 2007; Ruchin 2008b).

Materials. Temnikov Distr., Temnikov, 1.VI.2008, 2 ex.; Veselyi, 6.VI.2015, 1 ex.; Alkayevo, 15.VIII.2015, 1 ex.; MNSR, quarter 436, 13.V.2017, 1 ex.; cordon Dolgiy Most, 13.V.2017, 1 ex., Egorov L.V.; quarter 442, 3.VI.2017, 1 ex.; cordon Pavlovskiy, quarter 420, 16.VI.2017, 1 ex.; cordon Inorskiy, 23-24.VIII.2017, 1 ex., Semishin G.B.; quarter 436, 12.V.2018, 1 ex., Semishin G.B.; quarter 19, 15.V.2018, 1 ex., Egorov L.V.; quarter 37, 16.V.2018, 1 ex., Egorov L.V.; quarter 381, 28.V.2018, 1 ex.; quarter 37, 8.VII.2018, 1 ex. Torbeevo Distr., Vindrey, 6.VI.2008, 1 ex. Torbeevo Distr., Drakino, 26.VII.2016, 1 ex. Elniki Distr., Novoyamskaya Sloboda, 23.VII.2016, 1 ex. Krasnoslobodsk Distr., Penkovo, 31.VIII.2008, 1 ex. Kovylkino Distr., Andreevka, 30.VII.2008, 5 ex. Staroe Shaygovo Distr., Staroe Akshino, 11.V.2008, 16.VIII.2008, 5 ex.; Staroe Akshino, 30.VII.2017, 1 ex. Kadoshkino Distr., Latyshovka, 4.VI.2016, 1 ex. Insar Distr., Kochetovka, 4.VI.2016, 1 ex.; Vasina Polyana, 4.VI.2016, 1 ex.; Novley, 19.VIII.2017, 1 ex. Ichalki Distr., Khanineevka, 23.VII.2008, VI-VII.2009, 2 ex.; NPS, Barakhmanovskoe forestry, $2 \mathrm{~km} \mathrm{NE}$ of Tashkino, 6.IX.2009, 1 ex.; NPS, Lvovskoe forestry, quarter 53, 23.V.2017, 24.V.2017, 2 ex., Semishin G.B.; NPS, Barakhmanovskoe forestry, quarter 74, 13.IX.2017, 1 ex., Semishin G.B. Ardatovo Distr., Piksyasi, 10.VIII.2018, 1 ex. Romodanovo Distr., Pushkino, 17.VIII.2017, 4 ex. Ardatov Distr., Sosnovoe, 18.VIII.2008, 3 ex.; Svetotekhnika, 18.V.2008, 2 ex. Chamzinka Distr., Komsomolskiy, VI.2008, 1 ex., Ryzhov M.K.; Gorbunovka, 24.VI.2016, 1 ex.; Picheury, 28.VII.2018, 1 ex. Atyashevo Distr., Selishchi, 10.VIII.2017, 6 ex. Dubenki Distr., Yavleyka, 10.VIII.2017, 2 ex. Lyambir Distr., Ekaterinovka, 9.V.2008, 29.V.2008, 3 ex.; Belogorskoe, 29.VII.2016, 2 ex. Ruzaevka Distr., Levzhenskiy, 22.VII.2008, 28.VII.2016, 1.V.2016, 9.V.2016, 8 ex.; Yakovshchina, 26.VI.2016, 2 ex.; Khovanshchina, 12.VI.2017, 1 ex. Bolshie Berezniki Distr., 9 km S Simkino, 24.VI.2005, 1 ex., 20.V.2007, 1 ex.; Gart, 19.VII.2008, 1 ex.; Nerley, 5.VI.2016, 1 ex.; Permisi, 5.VI.2016, 1 ex.; Simkino, 12.VIII.2017, 2 ex.; Simkinskoe lesnichestvo, 12.VIII.2017, 2 ex. Kochkurovo Distr., Kachelay, 22.VII.2008, 2 ex., Kurmaeva D.K.; Starye 
Turdaki, 12.VI.2008, 1 ex.; Kochkurovo, 8.V.2016, 2 ex.; Novaya Pyrma, 11.VIII.2017, 1 ex.; Krasnaya Zorka, 29.VII.2017, 1 ex. Saransk, 6.VII.2007, 1 ex., 22.IV.2008, 16.V.2008, 10.V.2008, 21 ex.

Habitat. It is known in different biotopes (agrocoenoses, mixed, deciduous forests, floodplain meadows, steppe slopes, dry grasslands, abandoned lands, birch forests, forest plantations, wastelands, shores of water bodies. It was caught on Lathyrus sylvestris L., Padus avium Mill., Salix sp.

Harmonia quadripunctata (Pontoppidan, 1763)

Literature. Temnikov Distr. (Egorov and Ruchin 2014). Ichalki Distr. (Ruchin and Egorov 2013). Bolshie Berezniki Distr. (Ruchin et al. 2009b). Kochkurovo Distr. (Ruchin et al. 2009b). Saransk (Ruchin et al. 2009b).

Materials. Ichalki Distr., NPS, Barakhmanovskoe forestry, quarter 93, 4.VII.2017, 1 ex., Semishin G.B. Temnikov Distr., MNSR, quarter 86, 30.VIII.-27.IX.2018, 1 ex., Semishin G.B., Egorov L.V.

Habitat. It inhabits coniferous and mixed forests. The species individuals fly to the light.

*Oenopia conglobata (Linnaeus, 1758)

Materials. Temnikov Distr., MSNR, cordon Dolgiy Most, 13.V.2017, 1 ex., Egorov L.V.

Habitat. This species has been found in the glade along the mixed forest.

Myrrha octodecimguttata (Linnaeus, 1758)

Literature. Temnikov Distr. (Ruchin et al. 2009a; Feoktistov 2011; Egorov and Ruchin 2012).

Habitat. It has been found in glades along the mixed and pine forests, at forest edges.

\section{Sospita vigintiguttata (Linnaeus, 1758)}

Literature. Temnikov Distr. (Ruchin et al. 2009a; Feoktistov 2011; Egorov et al. 2015).

Materials. Temnikov Distr., MSNR, cordon Inorskiy, 13.V.2017, 1 ex., Egorov L.V.; MSNR, Pushta, 1.V.2018, 2 ex.; quarter 448, 19.V.2018, 1 ex., Egorov L.V.

Habitat. It inhabits floodplain meadows and mixed forests.

\section{Myzia oblongoguttata (Linnaeus, 1758)}

Literature. Temnikov Distr. (Redikortsev 1938; Ruchin et al. 2009a; Feoktistov 2011; Egorov and Ruchin 2014; Egorov et al. 2015, 2017).

Materials. Temnikov Distr., MSNR, Pushta, 14.VI.2017, 1 ex. Saransk, 4.VII.2007, 1 ex.

Habitat. It has been found in glades along mature pine forests with participation of spruce, birch, aspen (Populus tremula L.).

Anatis ocellata (Linnaeus, 1758) (Figure 1)

Literature. Temnikov Distr. (Ruchin et al. 2009a; Feoktistov 2011; Egorov and Ruchin 2012, 2013, 2014; Egorov et al. 2015, 2016).

Materials. Temnikov Distr., MSNR, quarter 19, 15.V.2018, 1 ex., Egorov L.V. Elniki Distr., Starye
Pichingushi, 25.VI.2012, 1 ex., Semishin G.B. Krasnoslobodsk Distr., Penkovo, 31.VIII.2008, 1 ex. Ichalki Distr., NPS, Kemlyanskoe forestry, Smolny, V.2009, 1 ex. Dubenki Distr., Nikolaevka, 27.VIII.2005, 1 ex. Lyambir Distr., Ekaterinovka, 2.VI.2009, 1 ex. Bolshie Berezniki Distr., 9 km S Simkino, 14.VII.1996, 1 ex. Saransk, 22.VI.2006, 1 ex.

Habitat. It has been found in glades, edges, narrow clearings in mixed and coniferous forests. The species individuals fly to the light.

Adalia bipunctata (Linnaeus, 1758) (Figure 1)

Literature. Temnikov, Krasnoslobodsk, Bolshie Berezniki, Ruzaevka districts (Red Data Book of the Republic of Mordovia 2005; Ruchin et al. 2007a; Timraleev at al. 2007). Saransk (Bieńkowski and OrlovaBienkowskaja 2008). Temnikov Distr. (Plavilshchikov 1964; Ruchin et al. 2009a; Egorov and Ruchin 2009b, 2012, 2013, 2014). Ichalki Distr. (Ruchin et al. 2007, 2008; Ruchin 2008b; Egorov and Ruchin 2009b). Lyambir Distr. (Egorov and Ruchin 2009b).

Materials. Temnikov Distr., MSNR, Pushta, 24.IV.2018, 4 ex., Semishin G.B.

Habitat. It has been found in floodplain meadows, steppe slopes, mixed and deciduous (including ones of secondary origin) forests, parks. The species is frequently found in high abundance during the wintering in houses. The species is often observed in heated wooden buildings and flats.

\section{Adalia decempunctata (Linnaeus, 1758)}

Literature. Temnikov Distr. (Plavilshchikov 1964; Egorov et al. 2017).

Materials. Bolshie Berezniki Distr., 9 km S Simkino, 23.VI.2005, 1 ex. Ichalki Distr., Khanineevka, VIVII.2009, 1 ex. Temnikov Distr., MSNR, Pushta, 17.V.2018, 1 ex. Zubova Polyana Distr., Yavas, 22.VI.2018, 1 ex.

Habitat. It inhabits deciduous and mixed forests.

Coccinella hieroglyphica Linnaeus, 1758

Literature. Temnikov Distr. (Ruchin et al. 2009a; Feoktistov 2011). Bolshoe Ignatovo Distr. (Ruchin 2008a). Ichalki Distr. (Ruchin et al. 2007; Ruchin 2008a, b). Bolshie Berezniki Distr. (Ruchin 2008a).

Materials. Staroe Shaygovo Distr., Lesnichestvo, 17.VII.2009, 1 ex. Ichalki Distr., NPS, Barakhmanovskoe forestry, $2 \mathrm{~km} \mathrm{NW}$ Tashkino, 6.IX.2009, 1 ex.; NPS, Lvovskoe forestry, quarter 53, 22.VI.2017, 1 ex., Semishin G.B. Kochkurovo Distr., Mordovskoe Davydovo, 23.VII.2008, 1 ex., Kurmaeva D.K. Temnikov Distr., MSNR, quarter 86, 20.IV.2018, 6 ex., Semishin G.B.

Habitat. It is known in floodplain meadows and mixed forests.

Coccinella magnifica L. Redtenbacher, 1843 (Figure 1)

Literature. Temnikov Distr. (Egorov et al. 2015, 2017). Ichalki Distr. (Egorov and Ruchin 2009a).

Materials. Temnikov Distr., MSNR, Pushta, 27.IV.2009, 1 ex. Zubova Polyana Distr., 5 km SW 
Bystrishchi, 1.V.2008, 1 ex. Bolshoe Ignatovo Distr., NPS, Aleksandrovskoe forestry, quarter 39, 11.VIII.2018, 1 ex.

Habitat. It has been found in glades of mixed and pine forests.

\section{Coccinella quinquepunctata Linnaeus, 1758}

Literature. Temnikov Distr. (Plavilshchikov 1964; Ruchin et al. 2009a; Egorov and Ruchin 2012, 2013). Romodanovo Distr., Ruzaevka Distr., Bolshie Berezniki Distr. (Antsiferova and Dobrosmyslov 1966; Antsiferova 1979). Ichalki Distr. (Ruchin et al. 2007; Ruchin 2008b). Ichalki Distr., Temnikov Distr., Chamzinka Distr., Zubova Polyana Distr., Kovylkino Distr., Torbeevo Distr., Ruzaevka Distr., Elniki Distr., Krasnoslobodsk Distr., Saransk, Staroe Shaygovo Distr., Ardatov Distr., Kochkurovo Distr. (Egorov and Ruchin 2009b).

Materials. Temnikov Distr., Alkaevo, 15.VIII.2015, 1 ex.; MSNR, Pushta, 23.VI.2016, 19.V.2018, 2 ex., Egorov L.V.; MSNR, quarter 427, 10.VIII.2016, 1 ex. Elniki Distr., Novoyamskaya Sloboda, 23.VIII.2016, 1 ex.

Habitat. It has been found in floodplain meadows, mixed forests and secondary deciduous forests, parks.

\section{Coccinella septempunctata Linnaeus, 1758}

Literature. Temnikov Distr. (Plavilshchikov 1964; Ruchin et al. 2009a; Egorov and Ruchin 2012, 2013, 2014; Egorov et al. 2015, 2016, 2017). Ichalki Distr. (Ruchin et al. 2007; Ruchin 2008b). Romodanovo Distr., Ruzaevka Distr., Bolshie Berezniki Distr. (Antsiferova et al. 1966; Antsiferova and Dobrosmyslov 1966; Antsiferova 1979).

Materials. Temnikov Distr., MSNR, quarter 354, 20.V.2017, 1 ex.; MSNR, quarter 403, 20.V.2017, 1 ex.; MSNR, quarter 111, 1.VIII.2017, 3 ex.; MSNR, quarter 413, 4.VIII.2017, 1 ex.; MSNR, quarter 397, 6.VIII.2017, 1 ex.; MSNR, quarter 86, 21.IV.-17.V.2018, 17.V.2018, 18.V.2018, 30.VIII.-27.IX.2018, 4 ex., Semishin G.B., Egorov L.V.; MSNR, quarter 35, 16.V.2018, 2 ex., Egorov L.V.; MSNR, quarter 383, 17.V.2018, 1 ex.; MSNR, quarter 368, 27.V.2018, 2 ex. Zubova Polyana Distr., 5 km SW Bystrishchi, 1.V.2008, 4 ex.; Tenishevo, 2.V.2008, 5 ex.; Mordovskaya Polyana, 7.VI.2008, 4 ex.; Udevo, 8.VI.2008, 3 ex.; Izvest, 8.VI.2008, 2 ex.; Vysha, 8.VI.2008, 1 ex. Aturyevo Distr., Chudinka, 7.VI.2008, 5 ex.; Pichepolonga, 2.VII.2016, 2 ex. Torbeevo Distr., Vindrey, 14.V.2008, 6.VI.2008, 6 ex.; Surgod, 21.VI.2016, 1 ex. Elniki Distr., Novye Shaly, 22.VII.2017, 1 ex. Elniki Distr., Malye Mordovskie Poshaty, 22.VII.2017, 1 ex. Krasnoslobodsk Distr., Krasnaya Podgora, 16.VII.2009, 2 ex. Kovylkino Distr., Novoe Mamangino, 6.V.2008, 3 ex. Staroe Shaygovo Distr., Staroe Akshino, 11.V.2008, 8 ex.; Staraya Terizmorga, 17.VII.2009, 1 ex.; Staroe Akshino, 30.VII.2017, 2 ex. Bolshoe Ignatovo Distr., NPS, Aleksandrovskoe forestry, quarter 39, 11.VIII.2018, 1 ex. Ichalki Distr., NPS, Barakhmanovskoe forestry, quarter 113, 28.VIII.-19.IX.2017, 3 ex., Semishin G.B. Ardatovo Distr., Piksyasi, 10.VIII.2018, 1 ex. Romodanovo Distr., Pushkino, 17.VIII.2017, 3 ex.; Zarechnyi, 3.VIII.2018, 1 ex. Ardatov Distr., Svetotekhnika, 18.V.2008, 11 ex.; 3 km N Sosnovoe, 18.VIII.2008, 1 ex. Chamzinka Distr., Kamenka, 6.IV.2008, 5 ex. Atyashevo Distr., Selishchi,
10.VIII.2017, 2 ex. Dubenki Distr., Yavleyka, 5.IV.2008, 10.VIII.2017, 5 ex.; Nikolaevka, 5.IV.2008, 7 ex. Insar Distr., Novley, 19.VIII.2017, 1 ex.; Verkhnyaya Lukhma, 19.VIII.2017, 1 ex. Lyambir Distr., Ekaterinovka, V.2007, 3 ex. Ruzaevka Distr., Levzhenskiy, 22.VII.2008, 3 ex.; 3 km N Streletskaya Sloboda, 19.V.2009, 1 ex. Bolshie Berezniki Distr., Gart, 5.IV.2008, numerous ex. Kochkurovo Distr., Starye Turdaki, 12.VI.2008, 4 ex.; Muran, 29.VII.2017, 2 ex.; Novaya Pyrma, 11.VIII.2017, 2 ex. Saransk, 15.V.2005, 23.V.2006, numerous ex.

Habitat. It has been found in different biotopes (agrocoenoses, mixed and deciduous forests, steppe slopes, dry grasslands, abandoned lands, forest plantations, wastelands, shores of water bodies). The species is observed in high abundance on cereal crops (Antsiferova 1978; Timraleev 1992) and on fodder bean crops. It depends directly on quantity of Aphidoidea (Antsiferova et al. 1966).

\section{- Coccinella undecimpunctata Linnaeus, 1758}

Remarks. Timraleev (1992) has noted it without indication of localities. It is not presented in the materials. This species is not presented in the materials from Mordovia. Its presence in Mordovia is highly unreliable. Hence, we recommend to exclude this species from the list of regional fauna.

\section{? Hippodamia septemmaculata (DeGeer, 1775)}

Remarks. Timraleev (1992) has noted it without indication of localities. This species is not presented in the materials from Mordovia. Its presence in Mordovia needs to be confirmed by modern data.

Habitat. According to literature (Isaev and Egorov 2006; Nikitsky and Ukrainsky 2016), this species is known nearby of water bodies, in wet meadows and wet forest edges.

\section{Hippodamia tredecimpunctata (Linnaeus, 1758)}

Literature. Temnikov Distr. (Plavilshchikov 1964; Ruchin et al. 2009a; Egorov and Ruchin 2012, 2013, 2014; Egorov et al. 2015, 2016, 2017). Ichalki Distr. (Ruchin et al. 2007; Ruchin 2008b).

Materials. Temnikov Distr., MSNR, quarter 424, 13.V.2017, 1 ex., Egorov L.V.; MSNR, quarter 442, 2.V.2018, 1 ex.; quarter 368, 27.V.2018, 1 ex.; MSNR, quarter 368, 18.VI.2018, 1 ex.; MSNR, quarter 19, 19.VI.2018, 1 ex., Semishin G.B. Zubova Polyana Distr., Krutets, 17.VII.2008, 1 ex.; Kargashino, 29.VII.2009, 1 ex.; Tenishevo, 2.VIII.2015, 1 ex. Elniki Distr., Starye Pichingushi, 16.VIII.2008, 1 ex., Kurmaeva D.K. Staroe Shaygovo Distr., Staroe Akshino, 11.V.2008, 1 ex. Ichalki Distr., NPS, Barakhmanovskoe forestry, $2 \mathrm{~km} \mathrm{NE}$ of Tashkino, 6.IX.2009, 1 ex.; NPS, Barakhmanovskoe forestry, quarter 74, 13.IX.2017, 1 ex., Semishin G.B.; NPS, Kemlyanskoe forestry, quarter 22, 11.VIII.2018, 1 ex. Kochkurovo Distr., Sabaevo, 3.IX.2009, 1 ex. Romodanovo Distr., Pushkino, 17.VIII.2017, 1 ex. Saransk, 3.VII.2004, 1 ex.

Habitat. In our expedition, we have caught this species in riverbanks and lakeshores, wet glades of mixed and 
deciduous forests, floodplain meadows. The species individuals fly to the light.

\section{Hippodamia variegata (Goeze, 1777)}

Literature. Temnikov Distr. (Plavilshchikov 1964; Ruchin et al. 2009a; Egorov and Ruchin 2012, 2014; Egorov et al. 2015, 2016, 2017). Ichalki Distr. (Ruchin et al. 2007; Ruchin 2008b).

Materials. Temnikov Distr., MSNR, quarter 368, 18.V.2018, 1 ex., Egorov L.V.; Karaevo, 25.V.1984, 1 ex., Volkov O.G.; Polyanki, 24.VII.2016, 1 ex. Zubova Polyana Distr., Krutets, 17.VII.2008, 1 ex.; Tenishevo, 2.VIII.2015, 1 ex. Aturyevo Distr., Pichepolonga, 2.VII.2016, 1 ex. Torbeevo Distr., Surgod, 21.VI.2016, 1 ex.; Drakino, 26.VII.2016, 3 ex. Elniki Distr., Malye Mordovskie Poshaty, 23.VII.2016, 1 ex.; Svobodnyi, 31.VII.2008, 1 ex.; Novoyamskaya Sloboda, 23.VII.2016, 2 ex. Kovylkino Distr., Andreevka, 30.VII.2008, 1 ex. Staroe Shaygovo Distr., Ingener-Pyatina, 10.VII.2016, 2 ex.; Konopat, 9.VII.2016, 1 ex.; Govorovo, 10.VII.2016, 1 ex. Chamzinka Distr., Gorbunovka, 24.VI.2016, 1 ex. Atyashevo Distr., Kamenka, 24.VI.2016, 1 ex.; Atyashevo, 24.VI.2016, 1 ex. Lyambir Distr., Atemar, 7.V.2016, 1 ex.; Novaya Uda, 29.VII.2016, 1 ex.; Belogorskoe, 29.VII.2016, 3 ex. Insar Distr., Verkhnyaya Lukhma, 19.VIII.2017, 2 ex. Ruzaevka Distr., Levzhenskiy, 28.VII.2016, 2 ex.; Palaevka, 26.VI.2016, 1 ex. Bolshie Berezniki Distr., Gart, 6.VII.2007, 1 ex.; 9 km S Simkino, 29.IX.2007, 1 ex. Kochkurovo Distr., Kachelay, 22.VII.2008, 14.VII.2018, 2 ex., Kurmaeva D.K.; Mordovskoe Davydovo, 23.VII.2008, 1 ex., Kurmaeva D.K.; Mordovskoe Davydovo, 25.VI.2016, 1 ex.; Sabaevo, 3.IX.2009, 1 ex.; Starye Turdaki, 8.IX.2009, 1 ex.; 5 km east of Sabaevo, 25.VI.2016, 1 ex.; Krasnaya Zorka, 4.VIII.2018, 1 ex. Saransk, 25.V.2007, 14.VII.2008, 2 ex.

Habitat. It is known in different open biotopes (birch forest glades, mixed and deciduous forests, mature pine forests with participation of spruce, birch, floodplain and dry meadows, steppe grasslands, abandoned lands, forest plantations). The species individuals fly to the light.

\section{Ceratomegilla notata (Laicharting, 1781) (Figure 1)}

Literature. Temnikov Distr. (Redikortsev 1938; Ruchin et al. 2009a; Egorov and Ruchin 2012, 2013, 2014; Egorov et al. 2015, 2016, 2017). Ichalki Distr. (Ruchin et al. 2007; Ruchin 2008b).

Materials. Tengushevo Distr., Barashevo, V.2008, 1 ex.; 6 km west of Barashevo, 18.VII.2015, 1 ex. Temnikov Distr., MSNR, cordon Dolgiy Most, 13.V.2017, meadow, 1 ex., Egorov L.V.; MSNR, cordon Inorskiy, 1-3.VI.2017, 1 ex., Semishin G.B.; MSNR, cordon Inorskiy, 13.V.2017, 14.V.2018, 29.V.2018, 4 ex., Egorov L.V., Semishin G.B.; MSNR, cordon Inorskiy, 21.V.2017, 1 ex.; MSNR, quarter 442, 3.VI.2017, 1 ex.; MSNR, cordon Drozhdenovskiy, 4.VI.2017, 1 ex.; MSNR, quarter 437, 28.VI.2017, 1 ex.; MSNR, Pushta, 19.V.2018, 1 ex., Egorov L.V.; MSNR, quarter 19, 15.V.2018, 1 ex., Egorov L.V.; MSNR, quarter 37, 16.V.2018, 3 ex., Egorov L.V.; MSNR, quarter 420, 18.V.2018, 1 ex., Egorov L.V.; MSNR, quarter 368, 18.V.2018, 2 ex., Egorov L.V.; MSNR, quarter 435,
14.VI.2018, 2 ex.; MSNR, quarter 434, 22.VII.2018, 2 ex. Zubova Polyana Distr., Udevo, 8.VI.2008, 1 ex. Torbeevo Distr., Vindrey, 6.VI.2008, 4 ex. Elniki Distr., Malye Mordovskie Poshaty, 23.VII.2016, 1 ex.; Cherlyay, 23.VII.2016, 1 ex. Krasnoslobodsk Distr., Staroe Sindrovo, 1.VIII.2008, 1 ex. Bolshoe Ignatovo Distr., NPS, Aleksandrovskoe forestry, Lesnoy, V.2007, 1 ex.; $4 \mathrm{~km} \mathrm{~S}$ Barakhmany, 22.V.2008, 2 ex. Ichalki Distr., Sosnovka, 6.VIII.2008, 1 ex.; NPS, Barakhmanovskoe forestry, $2 \mathrm{~km}$ NE of Tashkino, 6.IX.2009, 1 ex.; NPS, Lvovskoe forestry, quarter 53, 23.V.2017, 24.V.2017, 7.VI.2017, 6 ex., Semishin G.B. Chamzinka Distr., Komsomolskiy, VII.2008, 1 ex., Ryzhov M.K. Ardatov Distr., Turgenevo, 19.V.2008, 2 ex. Lyambir Distr., Ekaterinovka, 29.V.2008, 1 ex., 11.V.2009, 2.VI.2009, 4 ex. Bolshie Berezniki Distr., Simkinskoe forestry, 12.VIII.2017, 1 ex.; 5 km SE Permisi, 24.VII.2008, 1 ex., Kurmaeva D.K. Kochkurovo Distr., Kachelay, 3.IX.2009, 1 ex.

Habitat. It has been found in different wet biotopes (mixed and deciduous forests: linden Tilia, birch forests, floodplain meadows, shores of water bodies).

\section{Ceratomegilla undecimnotata (D.H. Schneider, 1792)}

Literature. Timraleev (1989) has noted it without indication of localities.

Materials. Bolshie Berezniki Distr., Simkino, 12.VIII.2017, 1 ex. Atyashevo Distr., Selishchi, 30.VI.2018, 1 ex.

Habitat. It has been found once on a steppe slope.

\section{Halyzia sedecimguttata (Linnaeus, 1758)}

Literature. Temnikov Distr. (Ruchin et al. 2009a; Feoktistov 2011; Egorov et al. 2015, 2017). Ichalki Distr. (Ruchin et al. 2007; Ruchin 2008a,b).

Materials. Temnikov Distr., MSNR, quarter 19, 15.V.2018, 2 ex., Egorov L.V.; MSNR, quarter 37, 16.V.2018, 2 ex., Egorov L.V.; MSNR, quarter 86, 17.V.2018, 1 ex., Egorov L.V. Zubova Polyana Distr., Mordovskaya Polyana, 7.VI.2008, 1 ex. Torbeevo Distr., Drakino, 26.VI.2017, 1 ex. Ichalki Distr., NPS, Barakhmanovskoe forestry, quarter 74, 13.IX.2017, 1 ex., Semishin G.B. Chamzinka Distr., Komsomolskiy, VII.2008, 1 ex., Ryzhov M.K. Insar Distr., Novley, 27.V.2017, 1 ex. Ruzaevka Distr., Yakovshchina, 26.VI.2016, 1 ex.; Russkoe Baymakovo, 29.IV.2017, 1 ex. Saransk, 10.VI.2008, 1 ex.

Habitat. It is known in immature pine forests with participation of birch, mixed forests, but mostly in deciduous forests of different origins. In addition, it has been found in meadows. The species individuals fly to the light.

\section{Psyllobora vigintiduopunctata (Linnaeus, 1758)}

Literature. Temnikov Distr. (Redikortsev 1938; Ruchin et al. 2009a; Egorov and Ruchin 2012, 2013, 2014; Egorov et al. 2015, 2016, 2017). Ichalki Distr. (Ruchin 2008b). Saransk (Botanical Garden of the Mordovia State University) (Timraleev 1989).

Materials. Temnikov Distr., Temnikov, 1.VI.2008, 2 ex.; MSNR, cordon Drozhdenovskiy, 18.VI.2017, 1 ex.; 
MSNR, cordon Inorskiy, 26.VII.2017, 3 ex., Semishin G.B.; MSNR, quarter 35, 16.V.2018, 1 ex., Egorov L.V.; MSNR, quarter 19, 17.V.2018, 1 ex., Egorov L.V.; MSNR, quarter 34, 28.VI.-17.VII.2018, 1 ex., Semishin G.B., Egorov L.V. Zubova Polyana Distr., Vysha, VI.2008, 1 ex.; Vadovo-Sosnovka, 30.VII.2009, 1 ex. Aturyevo Distr., Chudinka, 7.VI.2008, 1 ex. Torbeevo Distr., Vindrey, 6.VI.2008, 1 ex. Elniki Distr., Novye Shaly, 19.VII.2015, 1 ex.; Novoyamskaya Sloboda, 23.VII.2016, 1 ex. Krasnoslobodsk Distr., Penkovo, 31.VIII.2008, 1 ex.; Krasnoslobodsk, 5.VI.2009, 1 ex. Kovylkino Distr., Andreevka, 30.VII.2008, 1 ex. Staroe Shaygovo Distr., Staroe Akshino, 16.VIII.2008, 2 ex.; Staroe Akshino, 30.VII.2017, 1 ex.; Ingener-Pyatina, 10.VII.2016, 1 ex. Bolshoe Ignatovo Distr., NPS, Aleksandrovskoe forestry, Lesnoy, 6.V.2007, 1 ex. Ichalki Distr., NPS, Lvovskoe forestry, quarter 53, 23.V.2017, 7.VI.2017, 22.V.2017, 24.V.2017, 7.VII.2017, 6 ex., Semishin G.B. Ardatov Distr., Ardatov, 6.VII.2003, 1 ex.; Oktyabrskiy, 26.V.2017, 1 ex. Chamzinka Distr., Komsomolskiy, VII.2008, 1 ex., Ryzhov M.K. Atyashevo Distr., Kamenka, 24.VI.2016, 1 ex. Lyambir Distr., Ekaterinovka, V.2008, 1 ex.; Atemar, 7.V.2016, 2 ex.; Belogorskoe, 29.VII.2016, 1 ex.; Novaya Uda, 24.VI.2017, 1 ex. Insar Distr., Kochetovka, 4.VI.2016, 2 ex.; Vasina Polyana, 4.VI.2016, 1 ex.; Verkhnyaya Lukhma, 19.VIII.2017, 1 ex.; Novley, 27.V.2017, 1 ex. Ruzaevka Distr., Levzhenskiy, 5.V.2004, 9.V.2016, 28.VII.2016, 4 ex.; Yakovshchina, 26.VI.2016, 1 ex.; 3 km N Streletskaya Sloboda, 19.V.2009, 1 ex.; Khovanshchina, 12.VI.2017, 1 ex. Bolshie Berezniki Distr., 9 km S Simkino, 4.VII.2005, 20.V.2007, 2 ex.; Gart, 19.VII.2008, 1 ex. Kochkurovo Distr., Kachelay, 22.VII.2008, 1 ex., Kurmaeva D.K.; Starye Turdaki, 12.VI.2008, 2 ex.; Kochkurovo, 8.V.2016, 2 ex.; Podlesnaya Tavla, 8.V.2016, 1 ex.

Habitat. It is known in different biotopes (steppe slopes, agrocenoses, mixed, deciduous forests, floodplain and dry meadows, abandoned lands, forest plantations, wastelands).

Vibidia duodecimguttata (Poda, 1761)

Literature. Temnikov Distr. (Egorov and Ruchin 2014).

Materials. Chamzinka Distr., Komsomolskiy, VIII.2009, 1 ex., Ryzhov M.K. Dubenki Distr., Yavleyka, 11.VI.2017, 1 ex.

Habitat. It has been found in mixed forests and steppeficated sites with carbonate outcrops. The species individuals fly to the light.

\section{Coccidulinae Mulsant, 1846}

Coccidula rufa (Herbst 1783)

Literature. Temnikov Distr. (Egorov and Ruchin 2012; Egorov et al. 2015, 2016).

Habitat. It is known in semiaquatic habitats.

\section{Scymninae Mulsant, 1846}

* Nephus bipunctatus (Kugelann, 1794)

Materials. Temnikov Distr., MSNR, cordon Inorskiy, 12.V.-1.VI.2017, 1 ex., Egorov L.V., Semishin G.B.

Habitat. It was caught once in the mixed forest using window traps.

Nephus redtenbacheri (Mulsant, 1846)

Literature. Temnikov Distr. (Egorov and Ruchin 2012; Egorov et al. 2015).

Habitat. It is known in wet glades of pine forests with participation of spruce, birch.

\section{Scymnus ferrugatus (Moll, 1785)}

Literature. Temnikov Distr. (Egorov and Ruchin 2013, 2014; Egorov et al. 2015, 2016). Staroe Shaigovo Distr. (Ruchin et al. 2009b).

Materials. Temnikov Distr., MSNR, cordon Inorskiy, 13.V.2017, 2 ex., Egorov L.V.; MSNR, Pushta, 19.V.2018, 1 ex., Egorov L.V. Ichalki Distr., NPS, Lvovskoe forestry, quarter 53, 24.V.2017, 8 ex., Semishin G.B.

Habitat. It is known in glades of pine and deciduous forests (especially in birch forests), in floodplain meadows. This species is often caught at the individuals of Padus avium.

\section{Scymnus frontalis (Fabricius, 1787)}

Literature. Temnikov Distr. (Egorov and Ruchin 2014; Egorov et al. 2015).

Materials. Bolshie Berezniki Distr., Simkino, 1.VII.2008, M.Ya. \& A.O. Bieńkovski - 1 female (the collection of A. Beńkovski), A.S. Ukrainskiy det., 2013.

Habitat. It has been found in mixed forest edges, steppe cretaceous slopes.

Scymnus haemorrhoidalis Herbst, 1797

Literature. Temnikov Distr. (Egorov and Ruchin 2012, 2013, 2014; Egorov et al. 2015, 2016).

Habitat. It is known in pine forests with participation of spruce and birch, mixed forests, along the shores of water bodies.

Scymnus nigrinus Kugelann, 1794

Literature. Temnikov Distr. (Egorov and Ruchin 2013; Egorov et al. 2015).

Materials. Temnikov Distr., MSNR, cordon Inorskiy, 12.V.-1.VI.2017, 1 ex., Egorov L.V., Semishin G.B.

Habitat. It is known only in the Mordovia State Nature Reserve - in edges of pine forests with participation of spruce, birch, aspen and in mixed forests.

Scymnus suturalis Thunberg, 1795

Literature. Temnikov Distr. (Egorov and Ruchin 2013).

Habitat. It has been found in forest edges. 


\section{Hyperaspidinae Mulsant, 1846}

\section{Hyperaspis campestris (Herbst, 1783)}

Literature. Ardatov Distr. (Ruchin et al. 2009b).

Habitat. It has been found once in the wet glade of mixed forest.

Hyperaspis concolor (Suffrian, 1843) (= inexpectata Guenther, 1959) 2013).

Literature. Temnikov Distr. (Egorov and Ruchin

Habitat. It has been found once in the pine forest with participation of spruce, birch, aspen.

\section{* Hyperaspis erythrocephala (Fabricius, 1787)}

Materials. Aturyevo Distr., Pichepolonga, 2.VII.2016, 1 ex. Elniki Distr., Novye Shaly, 19.VII.2015, 1 ex.

Habitat. It has been found in immature thinned-out pine forests with participation of birch.

\section{Hyperaspis pseudopustulata Mulsant, 1853}

Materials. Lyambir Distr. (Ruchin et al. 2009b).

Habitat. It has been found once in the birch forest.

\section{Hyperaspis reppensis (Herbst, 1783)}

Literature. Temnikov Distr. (Egorov and Ruchin 2012; Egorov et al. 2017).

Materials. Temnikov Distr., MSNR, cordon Inorskiy, 12.V.-1.VI.2017， 1-15.VI.2017， 15-29.VI.2017, 1327.VII.2017, 10 ex., Egorov L.V., Semishin G.B.; MSNR, quarter 19, 15.V.2018, 1 ex. Egorov L.V.; MSNR, quarter 34, 16.V.- 28.VI.2018, 2 ex., Semishin G.B., Egorov L.V.; MSNR, quarter 19, 27.VI.-12.VII.2018, 1 ex., Semishin G.B., Egorov L.V.

Habitat. It has been found in floodplain oak forests with participation of linden, mixed forests, and nearby of water bodies.

\section{Chilocorinae Mulsant, 1846}

\section{Platynaspis luteorubra (Goeze, 1777)}

Literature. Temnikov Distr. (Egorov and Ruchin 2012; Egorov et al. 2015). Lyambir Distr. (Ruchin 2008a).

Materials. Kochkurovo Distr., Kochkurovo, 8.V.2016, 1 ex.

Habitat. It has been found in mixed and pine forests with participation of spruce, birch, aspen, in glades and forest edges, floodplain meadows.

\section{Exochomus quadripustulatus (Linnaeus, 1758)}

Literature. Temnikov Distr. (Plavilshchikov 1964; Ruchin et al. 2009a; Egorov and Ruchin 2012; Egorov et al. 2017).

Materials. Temnikov Distr., Tatarskoe Karaevo, 20.IX.2008, 16.IV.2017, 2 ex.; MSNR, Pushta, 19.V.2018, 1 ex., Egorov L.V. Ichalki Distr., NPS, Barakhmanovskoe forestry, quarter 74, 11.IX.2017, 13.IX.2017, 3 ex., Semishin G.B.

Habitat. It has been found in pine forests with participation of spruce, birch.
- Parexochomus nigromaculatus (Goeze, 1777)

Remarks. Timraleev (1989) has noted it without indication of localities. This xerophilic species (Nikitsky and Ukrainsky 2016) is not presented in the materials. Its presence in Mordovia is highly unreliable. Hence, we recommend to exclude this species from the list of regional fauna.

Chilocorus bipustulatus (Linnaeus, 1758)

Literature. Temnikov Distr. (Ruchin et al. 2009a; Feoktistov 2011; Egorov and Ruchin 2012).

Habitat. It has been found in mixed and pine forests with participation of spruce, birch.

\section{Chilocorus renipustulatus (L.G. Scriba, 1791)}

Literature. Temnikov Distr. (Redikortsev 1938; Ruchin et al. 2009a; Egorov and Ruchin 2014; Egorov et al. 2015).

Materials. Temnikov Distr., MSNR, cordon Inorskiy, 12.V.-1.VI.2017, 29.VI.-13.VII.2017, 9-27.IX.2017, 13.V.2018, 4 ex., Egorov L.V., Semishin G.B.; MSNR, cordon Pavlovskiy, quarter 420, 9.IX.2017, 1 ex., Egorov L.V. MSNR, quarter 19, 15.V.2018, 29.VIII.-27.IX.2018, 5 ex., Semishin G.B., Egorov L.V.; MSNR, quarter 420, 18.V.2018, 1 ex., Egorov L.V.; MSNR, Pushta, 19.V.2018, 1 ex., Egorov L.V.; MSNR, quarter 448, 19.V.2018, 1 ex., Egorov L.V. Ichalki Distr., NPS, Lvovskoe forestry, Obrezki, 19.IV.2009, 1 ex.; Obrezki, 24.V.2017, 1 ex., Semishin G.B.; NPS, Barakhmanovskoe forestry, quarter 74, 13.IX.2017, 1 ex., Semishin G.B. Saransk, 24.V.2007, 12.V.2009, 2 ex.

Habitat. It has been found in glades of deciduous and mixed forests, in mature pine forests with participation of spruce, birch, along streams in spruce forests with participation of alder, birch, sometimes - nearby of water bodies.

As a result of the long-term studies conducted in the Republic of Mordovia, to date, the list of Coccinellidae fauna noted for the region is presented by 49 species. Three of them $(C$. sinuatomarginata, $C$. undecimpunctata, $P$. nigromaculatus) are recommended to be excluded from the regional fauna. The presence of one species needs to be proved by modern data. In recent years, three species $(N$. bipunctatus, $H$. erythrocephala, $O$. conglobata) have been registered for the first time in the Republic of Mordovia. Thus, nowadays Coccinellidae fauna of the Republic of Mordovia includes 46 species.

The obtained species number of fauna is comparable to fauna of other regions of European Russia. For instance, Coccinellidae fauna of Penza region consists of 38 species (Ukrainsky and Pronina 2012), 49 species in Chuvash Republic (Isaev and Egorov 2006; Egorov 2008, 2012, 2013, 2014a,b, 2016), 57 species in Ulyanovsk region (Isaev and Egorov 2006), 56 species in Samara region (Isaev and Egorov 2006), 62 species in Saratov region (Ukrainsky and Sazhnev 2012), 60 species in Moscow region (Nikitsky and Ukrainsky 2016), 35 species in Nizhny region (Anufriev and Volovik 1980; Anufriev and Potanin 1997), 42 species in Vladimir region (Anufriev and Volovik 1980; Semenov 2009), 46 species in Kirov region 
(Shernin 1974; Yuferev 2001), 56 species in Orenburg region (Khristina and Ukrainsky 2016), 43 species in Lipetsk region (Tsurikov 2009, 2018; Sazhnev and Aksenenko 2018).

\section{ACKNOWLEDGEMENTS}

The authors are grateful to D.K. Kurmaeva (Saransk), M.K. Ryzhov (Chamzinka, Mordovia), A.O. Bieńkowski, M.Ja. Orlova-Bienkowskaja (Moscow) for kindly shared materials to be processed.

\section{REFERENCES}

Anufriev GA., Volovik MG. 1980. The fauna of some Coleoptera groups in the Gorkiy region (Coleoptera: Coccinellidae, Cantharidae, Melyrididae). In: Terrestrial and aquatic ecosystems. Gorkiy. [Russian].

Anufriev GA, Potanin DV. 1997. The new addition to Coccinellidae fauna of the Nizhniy Novgorod region. (Insecta, Coleoptera, Coccinellidae) In: Terrestrial and aquatic ecosystem. Gorkiy. [Russian].

Antsiferova TA. 1978. The efficiency increase of bee pollination and biological protection of buckwheat through Phacelia sowing. In Ecological-faunistical investigations in Non-chernozem zone of the European part of the USSR. Vol. 1. Saransk. [Russian].

Antsiferova TA. 1979. Insect community of Alfalfa and its seed productivity. In: Ecological-faunistical investigations in Nonchernozem zone of the European part of the USSR. Vol. 2. Saransk. [Russian].

Antsiferova TA, Dobrosmyslov PA. 1966. Entomofauna of oats-vetchphacelium and oats-vetch mixtures in the Mordovian ASSR. In Ecological-faunistica relationships of selected groups of invertebrates and vertebrates. Mordovia Book Publishing House, Saransk. [Russian].

Antsiferova TA, Dobrosmyslov PA, Makarov AT. 1966. Selected data of entomofauna on fodder bean crops - Vicia faba L. In: Ecologicalfaunistica relationships of selected groups of invertebrates and vertebrates. Mordovia Book Publishing House, Saransk. [Russian].

Bieńkowski AO, Orlova-Bienkowskaja MJ. 2008. Records of protected Coleoptera species in the Republic of Mordovia. In: Rare animals of the Republic of Mordovia: materials for maintenance of Red Data Book of the Republic of Mordovia for 2008. Publisher of the Mordovia State University, Saransk. [Russian].

Booth R. 1991. A new genus and species of coccinellid (Coleoptera: Coccinellidae) predator on Rastrococcus spp. (Homoptera: Pseudococcidae) from India. Bull Entomol Res 81 (2): 147-150. DOI:10.1017/S000748530005121X.

Bouchard P, Smith ABT, Douglas H, Gimmel ML, Brunke AJ, Kanda K 2017. Biodiversity of Coleoptera. In: Insect Biodiversity: Science and Society. Volume 1. 2nd ed. John Wiley \& Sons Ltd., New York. DOI 10.1002/9781118945568.ch11.

Cheah CASJ, McClure MS. 2000. Seasonal synchrony of life cycles between the exotic predator, Pseudoscymnus tsugae (Coleoptera: Coccinellidae) and its prey, the hemlock woolly adelgid Adelges tsugae (Homoptera: Adelgidae). Agric For Entomol 2: 241-251. DOI: 10.1046/j.1461-9563.2000.00073.x

Chikhlyaev I, Ruchin A. 2014. The helminth fauna study of European common brown frog (Rana temporaria Linnaeus, 1758) in the Volga basin. Acta Parasitologica 59 (3): 459-471. DOI: 10.2478/s11686014-0268-5.

Chikhlyaev IV, Ruchin AB, Fayzulin AI. 2016. The helminth fauna study of European common toad in the Volga Basin. Nat Environ Pollut Technol 15 (3): 1103-1109.

Chursina MA, Ruchin AB. 2018a. A checklist of Bombyliidae (Diptera) from Mordovia, Russia and variation of wing shape in Bombylius species. Biodiversitas 19 (6): 2147-2156. DOI 10.13057/biodiv/d190622.
Chursina MA, Ruchin AB. 2018b. A checklist of Syrphidae (Diptera) from Mordovia, Russia. Halteres 9: 57-73. DOI: 10.5281 /zenodo. 1255874

Delucchi V. 1954. Pullus impexus (Muls.) (Coleoptera, Coccinellidae), a Predator of Adelges piceae (Ratz.) (Hemiptera, Adelgidae), with notes on its Parasites. Bull Entomol Res 45 (2): 243-278. DOI:10.1017/S0007485300027085.

Egorov LV. 2008. New and rare Coleoptera species of Chuvashian fauna (Insecta, Coleoptera). Vestnik of the Chuvash State Pedagogical University 2 (58): 83-86. [Russian]

Egorov LV. 2012. Some data concerning the Coleoptera fauna of the State Nature Reserve «Prisursky». Information 1. Scientific proceedings of the State Nature Reserve «Prisursky» 27: 20-35. [Russian].

Egorov LV. 2013. Towards insect fauna of steppe Protected Areas of the Chuvashian Republic. Scientific proceedings of the State Nature Reserve «Prisursky» 28: 52-62. [Russian].

Egorov LV. 2014. Some data concerning the Coleoptera fauna of the State Nature Reserve «Prisursky». Information 2. Scientific proceedings of the State Nature Reserve «Prisursky» 29: 53-80. [Russian].

Egorov LV. 2014. New data for Coleoptera fauna (Insecta, Coleoptera) of the Chuvashian Republic. Report 8. Proceedings of the Kazan Branch of the Russian Entomological Society 3: 12-18. [Russian].

Egorov LV. 2016. Some data concerning the Coleoptera fauna of the State Nature Reserve «Prisursky». Information 5. Scientific proceedings of the State Nature Reserve «Prisursky» 31: 69-114. [Russian].

Egorov LV, Ruchin AB. 2009a. Additions to Coleoptera fauna of National Park "Smolny" (Republic of Mordovia). Vestnik of the Chuvash State Pedagogical University 1 (61): 63-69. [Russian].

Egorov LV, Ruchin AB. 2009b. Status of some species of Coleoptera in the Red Data Book of the Republic of Mordovia. In: Rare animals of the Republic of Mordovia: materials for maintenance of Red Data Book of the Republic of Mordovia for 2009. Saransk: Publisher of the Mordovia State University. P. 21-39. [Russian].

Egorov LV, Ruchin AB B. 2012. Some data concerning the Coleoptera fauna of the Mordovia State Nature Reserve. Proceedings of the Mordovia State Nature Reserve 10: 4-57. [Russian].

Egorov LV, Ruchin AB. 2013. Some data concerning the Coleoptera fauna of the Mordovia State Nature Reserve. Report 2. Proceedings of the Mordovia State Nature Reserve 11: 133-192. [Russian].

Egorov LV, Ruchin AB. 2014. Some data concerning the Coleoptera fauna of the Mordovia State Nature Reserve. Report 3. Proceedings of the Mordovia State Nature Reserve 12: 26-78. [Russian].

Egorov LV, Ruchin AB, Semishin GB. 2015. Some data concerning the Coleoptera fauna of the Mordovia State Nature Reserve. Report 4. Proceedings of the Mordovia State Nature Reserve 14: 82-156. [Russian].

Egorov LV, Ruchin AB, Semishin GB. 2016. Some data concerning the Coleoptera fauna of the Mordovia State Nature Reserve. Report 5. Proceedings of the Mordovia State Nature Reserve 16: 293-364. [Russian].

Egorov LV, Ruchin AB, Semishin GB. 2017. Some data concerning the Coleoptera fauna of the Mordovia State Nature Reserve. Report 6. Proceedings of the Mordovia State Nature Reserve 18: 81-143. [Russian].

Egorov LV, Shapovalov AM. 2017. On the distribution of a poorly known longicorn beetle, Phymatodes abietinus Plavilstshikov et Lurie, 1960 (Coleoptera, Cerambycidae: Cerambycinae). Ent Rev 97: 353-356.

Evans EW. 1991. Intra versus interspecific interactions of ladybeetles (Coleoptera: Coccinellidae) attacking aphids. Oecologia 87: 401408.

Grebennikov KA. 2016. Study of biodiversity of nature reserves of Russia in the digital age: experience and perspectives. Nature Conservation Research 1 (2): 1-10. http://dx.doi.org/10.24189/ncr.2016.012.

Fasulati KK. 1971. Field studying of land invertebrates. Vol. 2. Vysshaya Shkola, Moscow. [Russian].

Félix S, Soares AO. 2004. Intraguild predation between the aphidophagous ladybird beetles Harmonia axyridis and Coccinella undecimpunctata (Coleoptera: Coccinellidae): the role of body weight. Eur J Entomol 101 (2): 237-242. DOI: 10.14411/eje.2004.032.

Feoktistov VF. 2011. The list of insect species discovered for the first time in the Mordovia State Nature Reserve and in adjacent territories. Mordovia University Bulletin 4: 83-89. [Russian].

Iablokoff-Khnzorian SM. 1983. A Review of the Family Coccinellidae (Coleoptera) of the Fauna of the USSR. Zoological Proceedings of the 
Institute of Zoology of the Academy of Science of Armenian SSR 19: 94-161. [Russian].

Iftikhar A, Aziz MA, Naeem M, Ahmad M, Mukhtar T. 2018. Effect of temperature on demography and predation rate of Menochilus sexmaculatus (Coleoptera: Coccinellidae) reared on Phenacoccus solenopsis (Hemiptera: Pseudococcidae). Pakistan J Zool 50 (5): 1885-1893. DOI: 10.17582/journal.pjz/2018.50.5.1885.1893.

Isaev AY, Egorov LV. 2006. Towards the fauna of selected groups of Cucujoidea (Coleoptera) superfamily of forest-steppe zone of Middle Volga Region. Report 1. The families Erotylidae, Endomychidae and Coccinellidae. Caucasian Entomological Bulletin 2 (1): 65-72. [Russian].

Kajita Y, Takano F, Yasuda H, Agarwala BK. 2000. Effects of indigenous ladybird species (Coleoptera: Coccinellidae) on the survival of an exotic species in relation to prey abundance. Appl Entomol Zool 35: 473-470. DOI: 10.1303/aez.2000.473.

Khan MH, Yoldas Z. 2018. Intraguild predation between two aphidophagous coccinellids, Hippodamia variegata (G.) and Coccinella septempunctata $\mathrm{L}$. (Coleoptera: Coccinellidae): the role of prey abundance. Biol Control 126: 7-14. DOI 10.1016/j.biocontrol.2018.07.011.

Khristina KA, Ukrainsky AS. 2016. The fauna of ladybird beetles (Coleoptera: Coccinellidae) of Orenburgskaya Oblast, Russia. Euroasian Entomol J 15 (3): 277-287.

Kovář I. 2007. Fam. Coccinellidae. In: I. Löbl, A. Smetana (eds): Catalogue of Palaearctic Coleoptera. Vol. 4. Elateroidea Derodontoidea - Bostrichoidea - Lymexyloidea - Cleroidea Cucujoidea. Apollo Books, Stenstrup.

Korotyaev BA. Lobanov AL. 2019. Family Coccinellidae Latreille, 1807 Ladybirds. Draft version of the list 07/01/2019. https://www.zin.ru/Animalia/Coleoptera/eng/cocc_ru.htm

Nikitsky NB, Ukrainsky AS. 2016. The Ladybird Beetles (Coleoptera, Coccinellidae) of Moscow Province. Entomol Rev 96 (6): 710-735.

Mizell RF. 2007. Impact of Harmonia axyridis (Coleoptera: Coccinellidae) on native arthropod predators in pecan and crape myrtle. Florida Entomol 90 (3): 524-536. DOI: 10.1653/0015-4040 (2007)90[524:IOHACC]2.0.CO;2.

Orlova-Bienkowskaja MJ, Bieńkowski AO. 2017. Alien Coccinellidae (Ladybirds) in Sochi National Park and its vicinity, Russia. Nature Conserv Res 2 (4): 96-101. DOI: 10.24189/ncr.2017.044.

Plavilshchikov NN. 1964. A list of insect species found in the Mordovian State Nature Reserve. Proceedings of the Mordovia State Nature Reserve 2: 105-134. [Russian].

Red Data Book of the Republic of Mordovia. 2005. Red Data Book of the Republic of Mordovia. Vol. 2: Animals. Publisher of the Mordovia State University, Saransk. [Russian].

Redikortsev VV. 1938. Materials to the entomofauna of the Mordovia State Nature Reserve. In: Fauna of the Mordovia State Nature Reserve: Scientific results of the zoological expedition under the guidance of Prof. S.S. Turov in 1936. Moscow. [Russian].

Ruchin AB. 2008a. New species of beetles (Coleoptera, Insecta) of the Republic of Mordovia. In: Organisms, Populations, Ecosystems: Problems and Ways of Biodiversity Conservation. Vologda. [Russian].

Ruchin AB. 2008b. List of insect species of National Park «Smolny». Proceedings of the National Park «Smolny» 1: 151-180. [Russian].

Ruchin AB. 2015. The additional list of insect species of National Park «Smolny». Proceedings of the National Park «Smolny» 2: 102-121. [Russian].

Ruchin AB. 2018. Biology and distribution of the Clouded Apollo Parnassius mnemosyne (Linnaeus, 1758) (Lepidoptera: Papilionidae), a rare butterfly in the Republic of Mordovia, Russia. Journal of Threatened Taxa 10 (7): 11980-11983. htp://doi.org/10.11609/jot. 3709.10.7.11980-11983

Ruchin AB, Antropov AV, Khapugin AA. 2019. Distribution, abundance, and habitats of rare species Parnopes grandior (Pallas 1771) (Hymenoptera, Chrysididae) in Mordovia and adjacent regions, Russia. Biodiversitas 20 (2): 303-310. DOI: 10.13057/biodiv/d200201

Ruchin AB, Artaev ON. 2016. On expansion of the distribution range of some scoliid wasps (Scoliidae, Hymenoptera, Insecta) in the Middle Volga region. Res J Pharmaceut Biol Chem Sci 7 (3): 2110-2115.

Ruchin AB, Egorov LV. 2013. New data on Coleoptera fauna of National Park «Smolny» (the Republic of Mordovia). University proceedings. Volga region 3 (3): 21-28. [Russian]

Ruchin AB, Egorov LV. 2017. Overview of insect species included in the Red Data Book of Russian Federation in the Mordovia State Nature
Reserve. Nature Conservation Research 2 (Suppl. 1): 2-9. DOI: 10.24189/ncr.2017.016 [Russian].

Ruchin AB, Egorov LV. 2018a. Discovery of Allonyx quadrimaculatus (Schaller, 1783) (Coleoptera Cleridae Clerinae) in Russia. Redia 101: 143-146. http://dx.doi.org/10.19263/REDIA-101.18.19.

Ruchin AB, Egorov LV. 2018b. Fauna of longicorn beetles (Coleoptera: Cerambycidae) of Mordovia. Russian Entomol J 27 (2): 161-177. DOI: 10.15298/rusentj.27.2.07.

Ruchin AB, Egorov LV. 2018c. Leptura aurulenta (Coleoptera, Cerambycidae), a new record of a very rare species in Russia. Nature Conservation Research 3 (1): 88-91. DOI: 10.24189/ncr.2018.003.

Ruchin AB, Egorov LV, Bugaev KE. 2009. New data of coleopterafauna of the Mordovia State Nature Reserve. In: XXIII Lyubishchev's Readings. Ulyanovsk. P. 409-416. [Russian].

Ruchin AB, Egorov LV, Alekseev SK, Kurmaeva DK, Ryzhov MK, Semishin GB. 2009. New species of beetles (Insecta: Coleoptera) in fauna of the Republic of Mordovia. Proceedings of the Kaluga Society for Study of Nature of the Local Area 9: 73-86. [Russian].

Ruchin AB, Egorov LV, Semishin GB. 2018. Fauna of click beetles (Coleoptera: Elateridae) in the interfluve of Rivers Moksha and Sura, Republic of Mordovia, Russia. Biodiversitas 19 (4): 1352-1365. DOI: $10.13057 /$ biodiv/d190423.

Ruchin AB, Grishutkin GF. 2018. Biology and distribution of Parnassius apollo (Linnaeus, 1758) a rare species in Mordovia Republic, Russia. Biodiversitas 19 (2): 431-436. DOI:10.13057/biodiv/d190210.

Ruchin AB, Grishutkin GF, Kurmaeva DK, Lapshin AS. 2008. Rare insect species of National Park «Smolny» and its buffer zone. Proceedings of the National Park «Smolny» 1: 181-186. [Russian].

Ruchin AB, Kurmaeva DK. 2010. On rare insects of Mordovia included in the Red Book of the Russian Federation. Entomol Rev 90: 712-717. DOI:10.1134/S0013873810060060

Ruchin AB, Kurmaeva DK, Spiridonov SH. 2007. New discovery and quantity of rare Invertebrata of the Republic of Mordovia (according to research of 2007.) In: Rare animals of the Republic of Mordovia: materials for maintenance of Red Data Book of the Republic of Mordovia for 2007. Publisher of the Mordovia State University, Saransk. [Russian].

Ruchin AB, Loginova NG, Kurmaeva DK. 2007. The entomofauna of two forestries of National Park «Smolny». In: Fauna and ecology of insects, Vol. 1. Publisher «Tsentr valeologii vuzov Rossii», Rostovon-Don. [Russian].

Ruchin AB, Makarkin NV. 2017. Neuroptera and Raphidioptera in the Mordovia State Nature Reserve. Nat Conserv Res 2 (2): 38-46. doi: 10.24189/ncr.2017.001 [Russian].

Ruchin AB, Mikhailenko AP. 2018. Fauna of mantids and orthopterans (Insecta: Mantodea, Orthoptera) of the Mordovia State Nature Reserve, Russia. Biodiversitas 19 (4): 1194-1206. DOI: 10.13057/biodiv/d190403.

Satti AA, Mahgoub HAH. 2018. Population abundance of Thrips tabaci Lindeman and its associated predators on some crops at Shendi, River Nile State, Sudan. International Journal of Scientific Progress and Research 54 (1): 37 - 45.

Sazhnev AS, Aksenenko EV. 2018. New records of Coccinellidae (Insecta: Coleoptera) for Tambov, Lipetsk and Oryol Provinces. Entomological and parasitological researches in the Volga region 15: 34-36.

Semenov VB. 2009. The annotated list of beetles (Insecta, Coleoptera) of Central Meshchera. KMK Scientific Press Ltd., Moscow. [Russian].

Shernin AI. 1974. Orders Coleoptera, Strepsiptera. In: Animal of Kirov region. Vol. 2. Kirov. [Russian].

Soares AO, Serpa A. 2007. Interference competition between ladybird beetle adults (Coleoptera: Coccinellidae): effects on growth and reproductive capacity. Population Ecology 49 (1): 37-43. https://doi.org/10.1007/s10144-006-0020-6

Soares AO, Borges I, Borges PAV, Labrie G, Lucas É. 2008. Harmonia axyridis: What will stop the invader? BioControl 53 (1): 127-145. https://doi.org/10.1007/s10526-007-9141-x

Timraleev ZA. 1989. The fauna of ladybirds in the Mordovia Republic. In: All-Union conference on cadastre and the registration of the animal world. Thesis of reports. Part IV. [Russian]

Timraleev ZA. 1992. Harmful and useful insects of grain crops of the South of the non-Chernozem zone of Russia. Saransk. [Russian]

Timraleev ZA, Kamenev AG, Bardin OD. 2007. Insects Of Mordovia. Part II. Coleoptera. Saransk. [Russian]

Tomaszewska W, Egorov LV, Ruchin AB, Vlasov DV. 2018. First record of Clemmus troglodytes (Coleoptera: Coccinelloidea, Anamorphidae) 
for the fauna of Russia. Nature Conservation Research 3 (3): 103-105. DOI: $10.24189 /$ ncr.2018.016.

Tsurikov MV. 2009. Beetles of the Lipetsk region. Voronezh. [Russian].

Tsurikov MV. 2018. Ecological and faunistic analysis of imago of Coleoptera of the Central Russian forest-steppe. Voronezh. [Russian]

Ukrainsky AS, Pronina IG. 2012. Addition to the list of ladybird beetles (Coleoptera: Coccinellidae) of Penza Oblast, Russia. Samarskaya
Luka: problems of Regional and Global Ecology 21 (3): 164-166. [Russian].

Ukrainsky AS, Sazhnev AS. 2012. New records of ladybird beetles (Coleoptera: Coccinellidae) for Saratov Province. III. Entomological and parasitological researches in the Volga region 10: 45-51. [Russian] 